EPJ Web of Conferences 45, 01128 (2013)

DOI: $10.1051 /$ epjconf/20134501128

(C) Owned by the authors, published by EDP Sciences, 2013

\title{
On the use of hypodermic needles in electrospray
}

\author{
N. Rebollo-Muñoz ${ }^{1, a}, J$ M. Montanero ${ }^{1}$, and A. M. Gañán-Calvo ${ }^{2}$ \\ 1 Department of Mechanical, Energetic and Material Engineering, University of Extremadura, Avda. de Elvas s/n, E-06006 \\ Badajoz, SPAIN \\ 2 Department of Aerospace Engineering and Fluid Mechanics, University of Seville, Camino de los Descubrimientos s/n, \\ E-41092 Sevilla, SPAIN
}

\begin{abstract}
We present an experimental analysis of electrospray where the standard feeding capillary was replaced with a hypodermic needle. Both the current intensity and the jet diameter were measured for a variety of leaky-dielectric liquids. The results obtained with a feeding capillary and a hypodermic needle of similar dimensions were compared. An increase of the liquid conductivity was measured by assuming that the electric current obeys the same scaling law in both configurations. That increase was likely due to the electrochemical reaction taking place in the needle tip.
\end{abstract}

\section{Introduction}

Understanding the effects of electric fields on capillary systems is of great importance in a number of applications including inkjet printing, spring coating, electrowetting, mass spectrometry, fabrication of microspheres of biological materials, direct handling of living cells, etc. One such application is electrospray [1], which constitutes a very useful atomization technique to produce a variety of capillary shapes. Many electrohydrodynamic phenomena arising in electrospray have been studied both theoretically and experimentally over the last decades. The so-called steady cone-jet mode of electrospray has attracted much attention due to its capability of producing steadily drops with diameters ranging from hundreds of microns down to a few nanometers [2].

In the cone-jet mode of electrospray, a thin jet tapers from the tip of a liquid meniscus which hangs from the end of a feeding capillary. As the injected flow rate decreases, this meniscus becomes a complex fluidic structure, which relies on the delicate balance between surface tension, viscosity, and electric forces [3,4]. The experiments show that there is a minimum value of the flow rate below which the cone-jet configuration becomes unstable. The diameter of the emitted jet decreases as the injected flow rate decreases. Therefore, the existence of such a critical flow rate limits the minimum size of the emitted jets.

The critical flow rate below which the cone-jet configuration becomes unstable depends essentially on the fluid properties. However, the geometrical configuration may also affect the flow stability under certain conditions. Several methods have been proposed to enhance the meniscus stability, and thus producing smaller jets. Senn et al. [5] introduced a very thin wire inside the liquid meniscus to stop the recirculation cell appearing for low flow rates, and, in this way, to stabilize the liquid ejection. Larriba and Fernández de la Mora [6] made use of concentric needles to form a compound electrospray at flow rates much

\footnotetext{
a e-mail: noeliarm@unex.es
}

smaller than those reached with the standard emitter. Another possibility is to replace the feeding capillary commonly used in electrospray with a hypodermic needle (Figure 1). In this way, the tapering meniscus is substituted by a much more stable liquid film. The film flows over the needle's outer surface towards the tip driven by the electric forces. The jet is emitted from a very small Taylor cone formed in the needle tip. This configuration has already been used in the pioneering experiments of Dole et al. [7] and Fenn et al. [8,9]. Lozano and Martínez-Sánchez [10] also used an externally wetted emitter made of a grooved tungsten wire electrochemically etched.

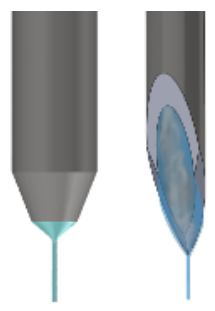

Fig. 1. Standard capillary (left) and needle emitter (right).

The electric field strength reaches very high values in the sharpened tip of a hypodermic needle. Therefore, electrochemical reactions not present in the standard electrospray configuration could take place when the feeding capillary is replaced with the needle. Those reactions could inject positive ions into the solution, and thus altering significantly its chemical composition. In that case, the liquid electric conductivity could considerably change too.

In this contribution, we present experimental results which show that the electrical conductivity significantly changes when electrospraying leaky-dielectric liquids with hypodermic needles. Both the current intensity and the jet diameter will be measured for a variety of liquids. The results obtained with a feeding capillary and a hypodermic needle of similar dimensions will be compared. If one as- 
sumes that the electric current obeys the same scaling law in both configurations, then one can measure the increase of the liquid conductivity owing to the electrochemical reaction taking place in the needle.

The results presented here must be regarded as preliminary. It would be desirable to measure the ionic composition of the spray emitted by the hypodermic needle to elucidate the role played by the electrochemical reaction in its tip.

\section{Experimental method}

The experimental setup is shown schematically in figure 2 . A liquid of density $\rho$, viscosity $\mu$, electrical conductivity $K$, and relative permittivity $\beta$ was injected at a constant flow rate $Q$ through a duct (A) by a syringe pump (Harvard Apparatus PHD 4400) connected to a stepping motor. The duct was either a cylindrical capillary of about 100 (110) $\mu \mathrm{m}$ in inner (outer) radius or a stainless steel hypodermic needle (Pic) of about 80 (150) $\mu \mathrm{m}$ in inner (outer) radius. The needle tip has an outer hydraulic radius $r_{t}$ of a few microns, and was chemically attacked with nitric acid to enhance the liquid capability of remaining attached to it. The capillary/needle was located at a distance of $1 \mathrm{~mm}$ from a metallic plate. The plate had an orifice (B) of $350 \mu \mathrm{m}$ in diameter located in front of the capillary/needle. The plate covered the upper face of a metallic cubic cell (C). We used a high-precision orientation system (D) and a translation stage (E) to ensure the correct alignment of these elements, and to set the capillary-to-orifice distance.

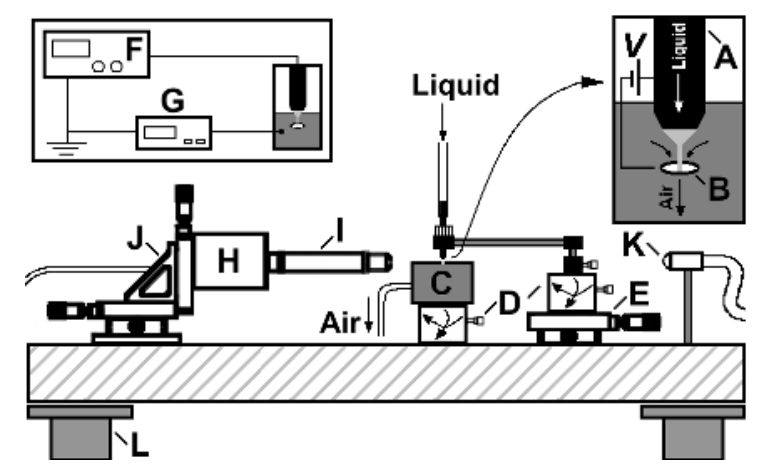

Fig. 2. Experimental setup: (A) needle/capillary, (B) plate with orifice, (C) suction cell, (D) orientation systems, (E) translation stage, $(\mathrm{F})$ high volatge power supply, $(\mathrm{G})$ picoamperimeter, $(\mathrm{H})$ camera, (I) optical lenses, (J) triaxial translation stage, (K) optical fiber connected to a stroboscope light source, and (L) antivibration isolation system.

An electric potential $V$ was applied to the end of the feeding capillary/needle through a DC high voltage power supply (Bertan 205B-10R) (F), and the cubic cell was used as ground electrode. A liquid meniscus was formed in the open air and stretched by the action of the electric field. Air can be regarded as a perfect dielectric gas of permittivity $\varepsilon_{o}$ equal to that of the vacuum. The air-liquid surface tension is $\sigma$.

A microjet of diameter $d_{j}$ tapered from the meniscus tip and moved vertically towards the plate orifice. A prescribed negative gauge pressure (about 10 mbar) was ap- plied in the cubic cell by using a suction pump to produce an air stream coflowing with the jet. Both the liquid jet and the coaxial air stream crossed the plate orifice, which prevented liquid accumulation on the metallic plate. In this way, all the electric charges were collected in the cubic cell. It must be noted that the mechanical effects associated with the air stream were negligible. In fact, the air speed was smaller than about $10 \%$ of that of the liquid at the orifice, and the density and pressure drops in the air stream were smaller than about $2 \%$. In addition, air ionization effects were less probable than in quiescent air because charges that could cause ionization cascades were flushed by the air stream.

Because the full scale of typical electric current measurements was in the nanoampere range, special care was taken for electrical shielding and grounding. The electric current $I$ transported by the liquid jet was measured using a picoamperimeter (Keithley model 6485) (G) connected to the cell.

Digital images of $1280 \times 960$ pixels were acquired using a CCD camera (Avt Stringay F-1125B) (H) equipped with optical lenses (an Optem HR 10X magnification zoomobjective and an Optem Zoom 70XL set of lenses with variable magnification from $0.75 \times$ to $5.25 \times$ ) (I) providing a frame covering an area of about $443 \times 332 \mu \mathrm{m}$. The magnification obtained was approximately $346 \mathrm{~nm} /$ pixel. The numerical aperture of the optical system was about 0.3 . The camera could be displaced both horizontally and vertically using a triaxial translation stage $(\mathrm{J})$ to focus the liquid meniscus. The fluid configuration was illuminated from the back by cool white light provided by an optical fiber (K) connected to a stroboscopic light source to reduce the image exposure time to about $3 \mu \mathrm{s}$. To check that the fluid configuration was axisymmetric, we also acquired images of the liquid meniscus using an auxiliary CCD camera (not shown in figure 2) with an optical axis perpendicular to that of the main camera. All these elements were mounted on an optical table with a pneumatic anti-vibration isolation system (L) to damp the vibrations coming from the building. By way of illustration, figure 3 shows the image of a jet produced by the experimental setup described above with a standard capillary (1-octanol, $Q=500 \mu \mathrm{l} / \mathrm{h}$ and $V=1.6$ $\mathrm{kV}$ ) and hipodermic needle (1-propanol, $Q=40 \mu \mathrm{l} / \mathrm{h}$ and $V=1.3 \mathrm{kV})$.
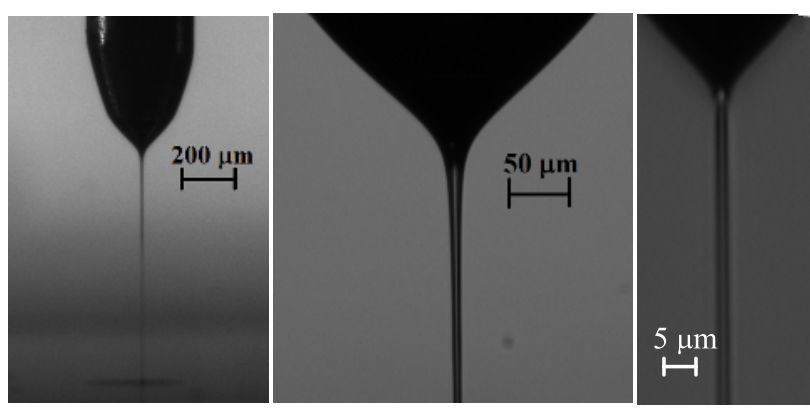

Fig. 3. 1-octanol jet (left and center) produced a standard capillary $(Q=500 \mu \mathrm{l} / \mathrm{h}$ and $V=1.6 \mathrm{kV})$. The left (center) image was acquired with the auxiliary (working) CCD camera. 1-propanol jet (right) produced with a hypodermic needle $(Q=40 \mu \mathrm{l} / \mathrm{h}$ and $V=1.3 \mathrm{kV})$. 
Table 1. Properties of the liquids used in the set of experimental series reported.

\begin{tabular}{|c|c|c|c|c|c|c|}
\hline Liquid & Symbol & $\rho\left(\mathrm{kg} \cdot \mathrm{m}^{-3}\right)$ & $\sigma\left(\mathrm{N} \cdot \mathrm{m}^{-1}\right)$ & $\beta$ & $\mathrm{K}\left(\mathrm{S} \cdot \mathrm{m}^{-1}\right)$ & $\mu(\mathrm{Pa} \cdot \mathrm{s})$ \\
\hline 1-octanol & $\triangle$ & 827 & 0.027 & 10 & $9.0 \times 10^{-7}$ & 0.0081 \\
1-decanol & $\nabla$ & 828 & 0.028 & 7.6 & $3.0 \times 10^{-7}$ & 0.012 \\
1-butanol & $\square$ & 800 & 0.023 & 17.8 & $6 \times 10^{-6}$ & 0.0025 \\
1-propanol & $\circ$ & 803 & 0.0237 & 20.3 & $9 \times 10^{-6}$ & 0.0019 \\
\hline
\end{tabular}

The properties of the working liquids are displayed in table 1 . The surface tension was determined using the Theoretical Image Fitting Analysis (TIFA) method [11]. The electrical conductivity was measured by applying a voltage difference between the ends of a cylindrical borosilicate capillary full of the working liquid, and then measuring the resulting electric current. The density, viscosity, and relative permittivity were taken from the manufacturer's specifications.

The contours of the free surface in the image were detected using a two-stage procedure. In the first stage, a set of pixels probably corresponding to the contour being sought was extracted using Otsu's method [12]. The accuracy of Otsu's method is limited to the pixel size. In the second stage, the accuracy of the detected contours was improved to the sub-pixel level by analyzing the gray intensity profile along the direction normal to the contour. Fitting the sigmoid (Boltzmann) function [13] to the gray intensity values allowed us to obtain a continuous function in the transient region of the gray profile. The contour point was found by applying the local thresholding criterion. The number of resulting contour points was approximately equal to the number of pixels of the image along the vertical direction. Once the left $x_{l}$ and right $x_{r}$ contours had been detected, they were rotated to their vertical position (the rotation angle was less than $2^{\circ}$ in all the cases analyzed), and the symmetry axis was found. Small non-axisymmetric perturbations of the free-surface were ignored by considering the axisymmetric contour $F=\left(x_{r}-\right.$ $\left.x_{l}\right) / 2$. Details of the image processing technique are given elsewhere $[14,15]$. Finally, the jet's diameter $d_{j}$ was obtained as the average of $2 F$ over a region where this quantity reached an almost constant value.

The experimental procedure consisted of the following steps. The capillary/needle was positioned in front of the metallic plate, and the air suction pump was switched on. The two cameras were positioned to focus the capillary/needle end. A constant liquid flow rate was injected through the capillary/needle by the syringe pump. The value of the applied voltage was selected to obtain the steady cone-jet mode. For a given liquid flow rate, there was a narrow range of applied voltage which yielded the formation of a Taylor cone. We selected the minimum value of that range. One of the cameras was displaced vertically to focus the region where the jet reached an almost constant radius. Finally, images of the jet were acquired, and the intensity current was measured with the picoamperimeter. In order to determine the minumum flow rate for a certain liquid, the above procedure was repeated by lowering progressively the flow rate.
Table 2. Minimum values of the flow rate measured in $\mu \mathrm{l} / \mathrm{h}$ for the standard feeding capillary and the hypodermic needle.

\begin{tabular}{|c|c|c|c|c|}
\hline & 1-octanol & 1-decanol & 1-butanol & 1-propanol \\
\hline Capillary & 500 & 400 & 400 & 400 \\
\hline Needle & 40 & 20 & 20 & 20 \\
\hline
\end{tabular}

\section{Results and discussion}

Table 2 shows the minimum values of the flow rate for which the steady cone-jet mode was obtained with the standard feeding capillary and the hypodermic needle. As can be observed, the minimum flow rates for the hypodermic needle are much lower than those of the standard feeding capillary, which shows the stabilizing role played by the needle.

Figure 4 shows the current intensity as a function of the liquid flow rate. The results were made dimensionless with the characteristic intensity $I_{0}=\varepsilon_{0}^{1 / 2} \sigma \rho^{-1 / 2}$ and flow rate $Q_{0}=\sigma \varepsilon_{0} /(\rho K)$. As expected, the results obtained with the standard feeding capillary match remarkably well the scaling law [16]

$$
I / I_{0}=2.6\left(Q / Q_{0}\right)^{1 / 2} .
$$

However, the current intensities measured with the hypodermic needle significantly exceed the theoretical prediction. This theoretical prediction is essentially based on the hypothesis that there is a transition in the tip of a Taylor cone from an electrostatic region where charges move due to Ohmic conduction to a tapering jet where charges are convected downstream. In principle, one can assume that this hypothesis also holds for the fluid configuration hanging from a hypodermic needle. If that were the case, the discrepancy between the experimental results and the scaling law (1) should be attributed to an increase of the liquid electrical conductivity.

Figure 5 shows the ratio of the modified electrical conductivity $K^{*}$ to its original value $K$. The quantity $K^{*}$ was calculated by fitting (1) to the experimental points measured with the hypodermic needle. As can be observed, there is a large increase of the liquid electric conductivity. This increase is assumed to be caused by the electrochemical reaction taking place in the needle tip, which injects positive charges into the liquid stream. Due to the low conductivity of the liquids used in the experiments, that charge injection resulted in an important increase of that property. We verified by simple optical inspection that the needle released a considerable amount of material in the course of the experiments. To simplify the analysis, we shall consider an only value for the modified electric conductivity of a certain liquid. That value is the average over those obtained for different flow rates. 


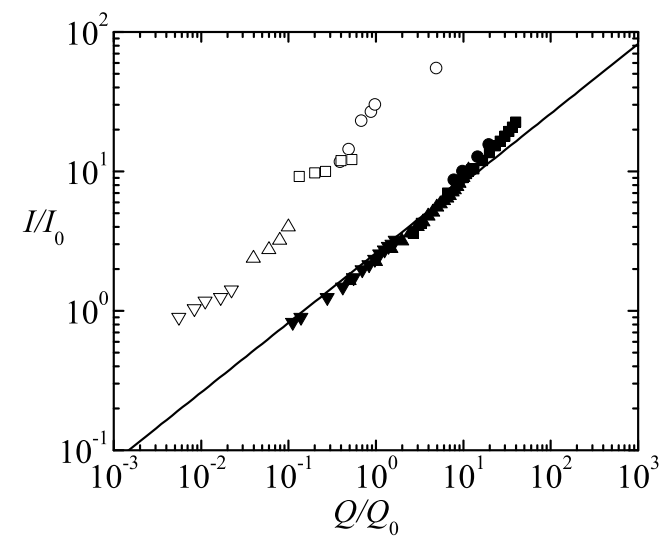

Fig. 4. Current intensity $I / I_{0}$ as a function of the flow rate $Q / Q_{0}$. The open and solid symbols correspond to the needle and capillary configurations, respectively. The solid line is the function (1).

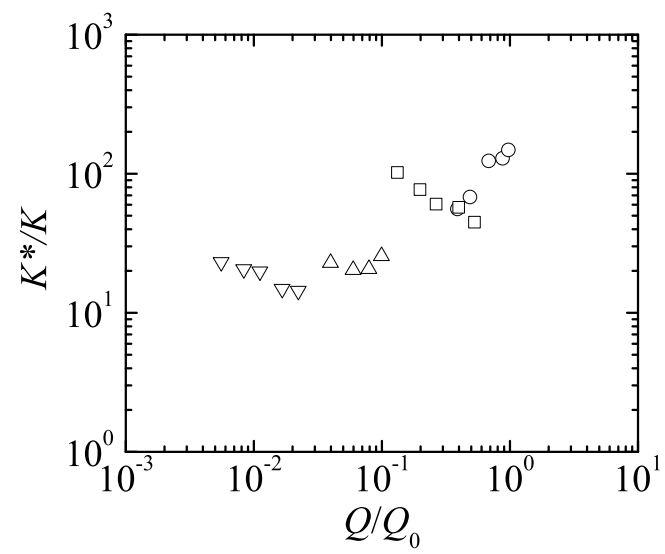

Fig. 5. Modified conductivity $K^{*} / K$ as a function of the flow rate $Q / Q_{0}$. The open and solid symbols correspond to the needle and capillary configurations, respectively.

Figure 6 shows again the current intensity versus de liquid flow rate. In this case, the characteristic flow rate $Q_{0}^{*}$ was calculated with the modified electrical conductivity. Hereafter, the symbol * means that the corresponding quantity was calculated with the original (modified) conductivity in the capillary (needle) case. Naturally, there is a reasonably good agreement between the scaling law and the experimental results obtained with both the capillary and the needle. Interestingly, the minimum values of $Q / Q_{0}^{*}$ are smaller for the experiments conducted with the standard feeding capillary. This result indicates that the stabilizing role played by the hypodermic needle must be explained in terms of the injection of positive charges, and not owing to a hydrodynamic effect.

Finally, the results obtained for the diameters of the emitted jets are plotted in figure 7 . The scaling law

$$
d_{j} / d_{0}^{*}=1.25\left(Q / Q_{0}^{*}\right)^{1 / 2}
$$

fits reasonably well the experimental points for the capillar. This law agrees with that obtained for the droplet diameter by Gañán-Calvo [16]. However, it fails to predict the jet diameter when the modified electrical conductivity is taken into account. The jet diameter seems to be sensitive to the geometrical configuration of the liquid source from which

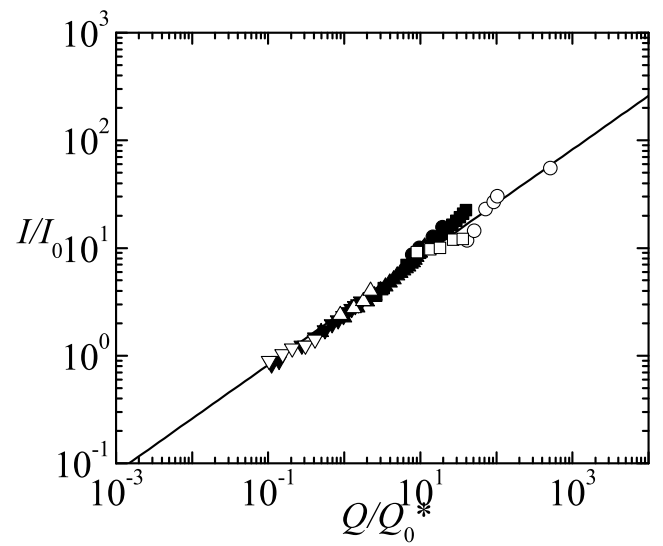

Fig. 6. Current intensity $I / I_{0}$ as a function of the flow rate $Q / Q_{0}^{*}$. The open and solid symbols correspond to the needle and capillary configurations, respectively. The solid line is the function (1).

the jet originates. It must be pointed out that the needle tip size and the jet radius are of the same order of magnitude, and, therefore, the former may determine to some extent the latter. One of the reasons why the experimental diameters exceed the theoretical prediction may be the energy dissipation due to the viscous friction between the film sliding over the needle's surface and that surface. A similar effect was observed in the flow focusing technique described in [17]. This point requires further investigation.

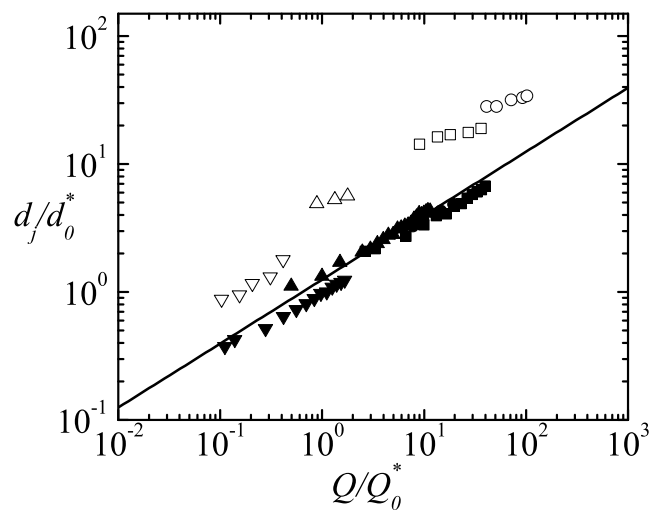

Fig. 7. Jet diameter $d_{j} / d_{0}^{*}$ as a function of the flow rate $Q / Q_{0}$. The open and solid symbols correspond to the needle and capillary configuration, respectively. The solid line is the function (2).

Partial support from the Ministry of Science and Education, Junta de Extremadura, and Junta de Andalucía (Spain) through Grants Nos. DPI2010-21103, GR10047, and P08-TEP-04128, respectively, is gratefully acknowledged.

\section{References}

1. J. Zeleny, Phys. Rev. 10, 1 (1917)

2. J.F. de la Mora, Annu. Rev. Fluid Mech. 39, 217 (2007)

3. A.M. Gañán-Calvo, J.M. Montanero, Phys. Rev. E 79, 066305 (2009) 
4. M.A. Herrada, J.M. López-Herrera, A.M. GañánCalvo, E.J. Vega, J.M. Montanero, S. Popinet, Phys. Rev. $E$ in press (2012)

5. A.K. Sen, J. Darabi, D.R. Knapp, J. Liu, J. Micromech. Microeng. 16, 620 (2006)

6. C. Larriba, J.F. de la Mora, Phys. Fluids 23, 102003 (2011)

7. M. Dole, L.L. Mack, R.L. Hines, R.C. Mobley, L.D. Ferguson, M.B. Alice, J. Chem. Phys. 49, 2240 (1968)

8. J.B. Fenn, M. Mann, C.K. Meng, S.F. Wong, C.M. Whitehouse, Science 246, 64 (1989)

9. C.M. Whitehouse, R.N. Dreyer, M. Yamashita, J.B. Fenn, Anal. Chem. 57, 675 (1985)

10. P. Lozano, M. Martínez-Sánchez, J. Phys. D: Appl. Phys. 38, 2371 (2005)

11. M.G. Cabezas, A. Bateni, J.M. Montanero, A.W. Neumann, Colloids Surf. A 255, 193 (2005)

12. N. Otsu, IEEE Transactions on Systems, Man, and Cybernetics (1979)

13. B. Song, J. Springer, J. Colloid Interface Sci. 184, 77 (1996)

14. J.M. Montanero, C. Ferrera, V.M. Shevtsova, Exp. Fluids 45, 1087 (2008)

15. E.J. Vega, J.M. Montanero, C. Ferrera, Measurement 44, 1300 (2011)

16. A.M. Gañán-Calvo, J. Aerosol Sci. 30, 863 (1999)

17. A.J. Acero, J.M. Montanero, C. Ferrera, A.M. GañánCalvo, J. Micromech. Microeng. submitted (2012) 